\title{
859.
}

\section{ON THE COMPLEX OF LINES WHICH MEET A UNICURSAL QUARTIC CURVE.}

[From the Proceedings of the London Mathematical Society, vol. xvII. (1886), pp. 232-238.]

- THE curve is taken to be that determined by the equations

$$
x: y: z: w=1: \theta: \theta^{3}: \theta^{4}
$$

viz. it is the common intersection of the quadric surface $\Theta=0$, and the cubic surfaces $P=0, Q=0, R=0$, where

$$
\Theta=x w-y z, \quad P=x^{2} z-y^{3}, \quad Q=x z^{2}-y^{2} w, \quad R=z^{3}-y w^{2} .
$$

Writing $(a, b, c, f, g, h)$ as the six coordinates of a line, viz.

$$
(a, b, c, f, g, h)=(\beta z-\gamma y, \gamma x-\alpha z, \alpha y-\beta x, \alpha w-\delta x, \beta w-\delta y, \gamma w-\delta z),
$$

if $(\alpha, \beta, \gamma, \delta),(x, y, z, w)$ are the coordinates of any two points on the line; then, if the line meet the curve, we have

$$
\begin{array}{r}
h \theta-g \theta^{3}+a \theta^{4}=0 \\
-h+f \theta^{3}+b \theta^{4}=0 \\
g-f \theta+c \theta^{4}=0 \\
-a-b \theta-c \theta^{3} \cdot=0
\end{array}
$$

from which four equations (equivalent, in virtue of the identity $a f+b g+c h=0$, to two independent equations), eliminating $\theta$, we have the equation of the complex. The form may, of course, be modified at pleasure by means of the identity-just referred to, but one form is

$$
\Omega,=a^{4}-b^{3} h+b f^{2} g+c g^{3}-a c f h+2 c^{2} h^{2}-4 a^{2} c h+a f^{3}-a^{3} f=0,
$$

as may be verified by substituting therein the values $a=-b \theta-c \theta^{3}, g=f \theta-c \theta^{4}$, $h=f \theta^{3}+b \theta^{4}$. The last-mentioned equation is thus the equation of the complex in question, in terms of the six coordinates $(a, b, c, f, g, h)$. 
If for the six coordinates we substitute their values, $\beta z-\gamma y$, \&c., we obtain $\Omega,=(x, y, z, w)^{4}(\alpha, \beta, \gamma, \delta)^{4}=0$, which, regarded as an equation in $(x, y, z, w)$, is the equation of the cone, vertex $(\alpha, \beta, \gamma, \delta)$, passing through the quartic curve; this equation should evidently be satisfied if only $\Theta, P, Q, R$ are each $=0$, viz. $\Omega$ must be a linear function of $(\Theta, P, Q, R)$; and by symmetry, it must be also a linear function of $\left(\Theta_{0}, P_{0}, Q_{0}, R_{0}\right)$, where

$$
\Theta_{0}=\alpha \delta-\beta \gamma, \quad P_{0}=\alpha^{2} \gamma-\beta^{3}, \quad Q_{0}=\alpha \gamma^{2}-\beta^{2} \delta, \quad R_{0}=\gamma^{3}-\beta \delta^{2},
$$

viz. the form is $\Omega,=(\Theta, P, Q, R)\left(\Theta_{0}, P_{0}, Q_{0}, R_{0}\right)$, an expression with coefficients which are of the first or second degree in $(x, y, z, w)$ and also of the first or second degree in $(\alpha, \beta, \gamma, \delta)$.

To work this out, I first arrange in powers and products of $(\alpha, \delta),(\beta, \gamma)$, expressing the quartic functions of $(x, y, z, w)$ in terms of $(\Theta, P, Q, R)$, as follows:

\begin{tabular}{|c|c|c|c|c|c|c|c|c|c|c|c|}
\hline & $a^{4}$ & $-b^{3} h$ & $+b f^{2} g$ & $+c g^{3}$ & $-a c f h$ & $+2 c^{2} h^{2}$ & $-4 a^{2} c h$ & $+a f^{3}$ & $-a^{3} f$ & & \\
\hline $\begin{array}{r}a^{4} \\
a^{3} \delta \\
a^{2} \delta^{2} \\
a \delta^{3} \\
\delta^{4} \\
a^{3} \beta \\
a^{2} \beta \delta \\
a \beta \delta^{2} \\
\beta \delta^{3} \\
a^{3} \gamma \\
a^{2} \gamma \delta \\
a \gamma \delta^{2} \\
\gamma \delta^{3} \\
a^{2} \beta^{2} \\
a^{2} \beta \gamma \\
a^{2} \gamma^{2} \\
a \beta^{2} \delta \\
\alpha \beta \gamma \delta \\
a \gamma^{2} \delta \\
\beta^{2} \delta^{2} \\
\beta \gamma \delta^{2} \\
\gamma^{2} \delta^{2} \\
a \beta^{3} \\
a \beta^{2} \gamma \\
a \beta \gamma^{2} \\
a \gamma^{3} \\
\beta^{3} \delta \\
\beta^{2} \gamma \delta \\
\beta \gamma^{2} \delta \\
\gamma^{3} \delta \\
\beta^{4} \\
\beta^{3} \gamma \\
\beta^{2} \gamma^{2} \\
\beta \gamma^{3} \\
\gamma^{4} \\
\end{array}$ & $\begin{array}{l}+z^{4} \\
-4 y z^{3} \\
+6 y^{2} z^{2} \\
-4 y^{3} z \\
+y^{4}\end{array}$ & $\begin{array}{l}-3 x z^{2} w \\
-3 x^{2} z^{2}\end{array}$ & $\begin{array}{l}-x y w^{2} \\
+2 x^{2} y w \\
+x w^{3} \\
-2 x^{2} w^{2} \\
+x^{3} w\end{array}$ & $\begin{array}{l}+3 y^{3} w \\
+x y^{3} \\
-x^{3} y \\
-3 y^{2} w^{2} \\
-3 x y^{2} w \\
+y w^{3}\end{array}$ & $\begin{array}{l}+y z^{2} w \\
-x y z^{2} \\
\\
-y^{2} z w \\
+x y z^{2} \\
\\
-y z w^{2} \\
+y^{2} w^{2} \\
-x z^{2} w \\
+2 x y z w \\
-x y^{2} w \\
+\quad x^{2} z^{2} \\
-x^{2} y z\end{array}$ & $\begin{array}{l}+2 y^{2} w^{2} \\
+8 x y z w \\
+2 x^{2} z^{2}\end{array}$ & $\begin{array}{l}-4 y z^{2} w \\
+8 y^{2} z w \\
-4 y^{3} w \\
-4 x z^{3} \\
+8 x y z^{2} \\
-4 x y^{2} z \\
\\
+4 x z^{2} w \\
-8 x y z w \\
+4 x y^{2} w\end{array}$ & $\begin{array}{l}+z w^{3} \\
-3 x z w^{2} \\
+3 x^{2} z w \\
-x^{3} z \\
-y w^{3} \\
+3 x y w^{2} \\
-3 x^{2} y w \\
+x^{3} y\end{array}$ & $\begin{array}{l}-z^{3} w \\
+3 y z^{2} w \\
-3 y^{2} z w \\
+y^{3} w \\
+x z^{3} \\
-3 x y z^{2} \\
+3 x y^{2} z \\
-x y^{3}\end{array}$ & 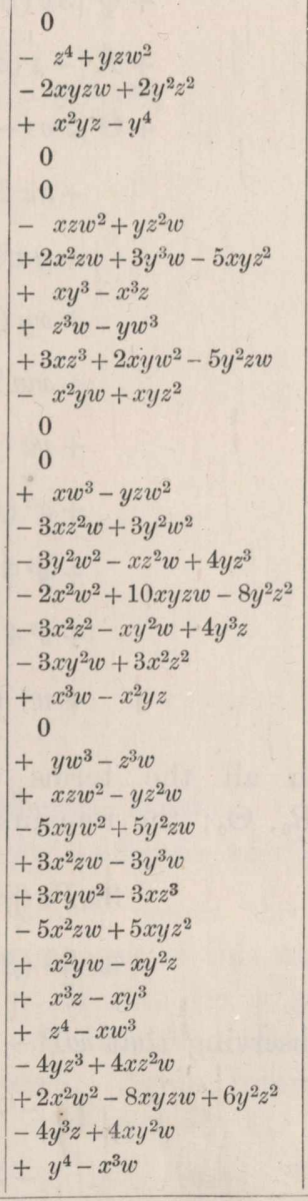 & $\begin{array}{l}-z R \\
-2 y z \Theta \\
+y P \\
\\
-\quad z w \Theta \\
+2 x z \Theta-3 y Q \\
-\quad x P \\
+w R \\
+2 y w \Theta+3 z Q \\
-\quad x y \Theta \\
\\
+\quad w^{2} \Theta \\
-3 w Q \\
-4 z^{2} \Theta+3 w Q \\
+(-2 x w+8 y z) \Theta \\
-4 y^{2} \Theta-3 x Q \\
+3 x Q \\
+\quad x^{2} \Theta \\
\\
-\quad w R \\
+z w \Theta \\
-5 y w \Theta \\
+3 w P \\
-3 x R \\
-5 x z \Theta \\
+x y \Theta \\
+x P \\
+\quad z R-w^{2} \Theta \\
+4 z^{2} \Theta \\
+(2 x w-6 y z) \Theta \\
+4 y^{2} \Theta \\
-y P-x^{2} \Theta\end{array}$ \\
\hline
\end{tabular}


Collecting the terms multiplied by $P, Q, R, \Theta$, respectively, we have

$$
\begin{aligned}
\Omega= & P\left\{y \alpha \delta^{3}-x \beta \delta^{3}+3 w \alpha \gamma^{3}+x \gamma^{3} \delta-y \gamma^{4}\right\} \\
+ & \left\{-3 y \alpha \beta \delta^{2}+3 z \alpha^{2} \gamma \delta-3 w \alpha^{2} \gamma^{2}+3 w \alpha \beta^{2} \delta-3 x \alpha \gamma^{2} \delta+3 x \beta^{2} \delta^{2}\right\} \\
+ & R\left\{-z \alpha^{3} \delta+w \alpha^{3} \gamma-w \alpha \beta^{3}-3 x \beta^{3} \delta+z \beta^{4}\right\} \\
+ & \left\{-2 y z \alpha^{2} \delta^{2}-z w \alpha^{2} \beta \delta+2 x z \alpha \beta \delta^{2}+2 y w \alpha^{2} \gamma \delta-x y \alpha \gamma \delta^{2}\right. \\
& +w^{2} \alpha^{2} \beta \gamma-4 z^{2} \alpha \beta^{2} \delta+(-2 x w+8 y z) \alpha \beta \gamma \delta-4 y^{2} \alpha \gamma^{2}+x^{2} \beta \gamma \delta^{2} \\
& +z w \alpha \beta^{2} \gamma-5 y w \alpha \beta \gamma \delta-5 x z \beta^{2} \gamma \delta+x y \beta \gamma^{2} \delta \\
& \left.-w^{2} \beta^{4}+4 z^{2} \beta^{3} \gamma+(2 x w-6 y z) \beta^{2} \gamma^{2}+4 y^{2} \beta \gamma^{3}-x^{2} \gamma^{4}\right\},
\end{aligned}
$$

which may be written as follows:-

$$
\begin{array}{rlr}
\Omega=P & \left\{y\left(\alpha \delta^{3}-\gamma^{4}\right)+x\left(\gamma^{3} \delta-\beta \delta^{3}\right)\right\} & +P\left(3 w \alpha \gamma^{3}\right) \\
+Q & \left\{3 x\left(\beta^{2} \delta^{2}-\alpha \gamma^{2} \delta\right)+3 w\left(\alpha \beta^{2} \delta-\alpha^{2} \gamma^{2}\right)\right\}+Q\left(3 z \alpha^{2} \gamma \delta-3 y \alpha \beta \delta^{2}\right) \\
+R & \left\{-z\left(\alpha^{3} \delta-\beta^{4}\right)+w\left(\alpha^{3} \gamma-\alpha \beta^{3}\right)\right\} & +R\left(-3 x \beta^{3} \delta\right) \\
+\Theta & \left\{z w\left(-\alpha^{2} \beta \delta+\alpha \beta^{2} \gamma\right)\right. & \\
& +x z 2\left(\alpha \beta \delta^{2}-\beta^{2} \gamma \delta\right) & \\
& +y w 2\left(\alpha^{2} \gamma \delta-\alpha \beta \gamma^{2}\right) & \\
& +x y\left(-\alpha \gamma \delta^{2}+\beta \gamma^{2} \delta\right) & \\
& +x w 2\left(-\alpha \beta \gamma \delta+\beta^{2} \gamma^{2}\right) & \\
& +y z\left(-2 \alpha^{2} \delta^{2}+8 \alpha \beta \gamma \delta-6 \beta^{2} \gamma \delta\right) \\
& +x^{2}\left(\beta \gamma \delta^{2}-\gamma^{4}\right) \\
& +y^{2} 4\left(-\alpha \gamma^{2} \delta+\beta \gamma^{2}\right) \\
& +z^{2} 4\left(-\alpha \beta^{2} \delta+\beta^{3} \gamma\right) \\
& +w^{2}\left(\alpha^{2} \beta \gamma-\beta^{4}\right)
\end{array}
$$

in which all the terms contained in the \{\} admit of expression in terms of $P_{0}, Q_{0}, R_{0}, \Theta_{0}$; the remaining six terms not included within $\{$. may be written

$$
\begin{gathered}
3 w P \alpha\left(\gamma^{3}-\beta \delta^{2}\right)+3(w P-y Q) \alpha \beta \delta^{2}-3 \Theta x z \beta^{2} \gamma \delta, \\
-3 x R \delta\left(\beta^{3}-\alpha^{2} \gamma\right)+3(-x R+z Q) \alpha^{2} \gamma \delta-3 \Theta y w \alpha \beta \gamma^{2}
\end{gathered}
$$

which, observing that $w P-y Q=x z \Theta$, and $-x R+z Q=y w \Theta$, are

$$
\begin{aligned}
& -3 w P \alpha\left(\gamma^{3}-\beta \delta^{2}\right)+3 x z \Theta\left(\alpha \beta \delta^{2}-\beta^{2} \gamma \delta\right), \\
& -3 x R \delta\left(\beta^{3}-\alpha^{2} \gamma\right)+3 y w \Theta\left(\alpha^{2} \gamma \delta-\alpha \beta \gamma^{2}\right) .
\end{aligned}
$$


The expression thus becomes

$$
\begin{aligned}
& \Omega=P . \quad x\left(\gamma^{3} \delta-\beta \delta^{3}\right) \\
& =x \delta R_{0} \\
& +y\left(\alpha \delta^{3}-\gamma^{4}\right) \\
& +3 w \alpha\left(\gamma^{3}-\beta \delta^{2}\right) \\
& =y\left(-\gamma R_{0}+\delta^{2} \Theta\right) \\
& =3 w \alpha R_{0} \\
& +Q .-3 x\left(\beta^{2} \delta^{2}-\alpha \gamma^{2} \delta\right) \\
& =-3 x \delta Q_{0} \\
& +3 w\left(\alpha \beta^{2} \delta-\alpha^{2} \gamma^{2}\right) \\
& =-3 w a Q_{0} \\
& +R .-3 x \delta\left(\beta^{3}-\alpha^{2} \gamma\right) \\
& =3 x \delta P_{0} \\
& -z\left(\alpha^{3} \delta-\beta^{4}\right) \\
& =z\left(-\beta P_{0}-\alpha^{2} \Theta_{0}\right) \\
& +w\left(\alpha^{3} \gamma-\alpha \beta^{3}\right) \\
& =w \alpha P_{0} \\
& +\Theta . \quad z w\left(-\alpha^{2} \beta \delta+\alpha \beta^{2} \gamma\right) \\
& =-z w \alpha \beta \Theta_{0} \\
& +5 x z\left(\alpha \beta \delta^{2}-\beta^{2} \gamma \delta\right) \\
& =5 x z \beta \delta \Theta_{0} \\
& +5 y w\left(\alpha^{2} \gamma \delta-\alpha \beta \gamma^{2}\right) \\
& =5 y w a \gamma \Theta_{0} \\
& +x y\left(-\alpha \gamma \delta^{2}+\beta \gamma^{2} \delta\right) \\
& =-x y \gamma \delta \Theta_{0} \\
& +2 x w\left(-\alpha \beta \gamma \delta+\beta^{2} \gamma^{2}\right) \\
& =-2 x w \beta \gamma \Theta_{0} \\
& +y z\left(-2 \alpha^{2} \delta^{2}+8 \alpha \beta \gamma \delta-6 \beta^{2} \gamma^{2}\right)=-2 y z(\alpha \delta-3 \beta \gamma) \Theta_{0} \\
& +x^{2}\left(\beta \gamma \delta^{2}-\gamma^{4}\right) \\
& =-x^{2} \gamma R_{0} \\
& +4 y^{2}\left(-\alpha \gamma^{2} \delta+\beta \gamma^{3}\right) \\
& =-4 y^{2} \gamma^{2} \Theta_{0} \\
& +4 z^{2}\left(-\alpha \beta^{2} \delta+\beta^{3} \gamma\right) \\
& =-4 z^{2} \beta^{2} \Theta_{0} \\
& +w^{2}\left(\alpha^{2} \beta \gamma-\beta^{4}\right) \\
& =w^{2} \beta P \text {; }
\end{aligned}
$$

and we thus finally obtain

$$
\begin{aligned}
\Omega= & P R_{0}(3 \alpha w-\gamma y+\delta x) \\
& +R P_{0}(3 \delta x-\beta z+\alpha w) \\
& +P \Theta_{0} \cdot \delta^{2} y \\
& +R \Theta_{0} \cdot-\alpha^{2} z \\
& +P_{0} \Theta \cdot \beta w^{2} \\
& +R_{0} \Theta \cdot-\gamma x^{2} \\
& -Q Q_{0} \cdot-3(\alpha w+\delta x) \\
& +\Theta \Theta_{0}\{-\alpha \beta z w-\gamma \delta x y+5 \beta \delta x z+5 \alpha \gamma y w-2 \beta \gamma x w-2 \alpha \delta y z \\
& \left.\quad-4 \gamma^{2} y^{2}+6 \beta \gamma y z-4 \beta^{2} z^{2}\right\},
\end{aligned}
$$

viz. $\Omega=0$ is the equation of the cone, vertex $(\alpha, \beta, \gamma, \delta)$, which passes through the quartic curve $x: y: z: w=1: \theta: \theta^{3}: \theta^{4}$. As regards the symmetry of this expression, it is to be remarked that, changing $(x, y, z, w)$ and $(\alpha, \beta, \gamma, \delta)$ into $(w, z, y, x)$ and $(\delta, \gamma, \beta, \alpha)$ respectively, we change $(\Theta, P, Q, R)$ and $\left(\Theta_{0}, P_{0}, Q_{0}, R_{0}\right)$ into $(\Theta,-R,-Q,-P)$ and $\left(\Theta_{0},-R_{0},-Q_{0},-P_{0}\right)$, respectively, and so leave $\Omega$ unaltered. Again, interchanging $(x, y, z, w)$ and $(\alpha, \beta, \gamma, \delta)$, we interchange $(\Theta, P, Q, R)$ and $\left(\Theta_{0}, P_{0}, Q_{0}, R_{0}\right)$, and so leave $\Omega$ unaltered. 